\title{
Developmental changes in visual responses to social interactions
}

\author{
Walbrin, Jon; Mihai, loana; Landsiedel, Julia; Koldewyn, Kami
}

\section{Developmental Cognitive Neuroscience}

\author{
DOI: \\ 10.1016/j.den.2020.100774
}

Published: 01/04/2020

Publisher's PDF, also known as Version of record

Cyswllt i'r cyhoeddiad / Link to publication

Dyfyniad o'r fersiwn a gyhoeddwyd / Citation for published version (APA):

Walbrin, J., Mihai, I., Landsiedel, J., \& Koldewyn, K. (2020). Developmental changes in visual responses to social interactions. Developmental Cognitive Neuroscience, 42, [100774].

https://doi.org/10.1016/j.dcn.2020.100774

\footnotetext{
Hawliau Cyffredinol / General rights

Copyright and moral rights for the publications made accessible in the public portal are retained by the authors and/or other copyright owners and it is a condition of accessing publications that users recognise and abide by the legal requirements associated with these rights.

- Users may download and print one copy of any publication from the public portal for the purpose of private study or research.

- You may not further distribute the material or use it for any profit-making activity or commercial gain

- You may freely distribute the URL identifying the publication in the public portal ?
}

Take down policy

If you believe that this document breaches copyright please contact us providing details, and we will remove access to the work immediately and investigate your claim. 


\title{
Developmental changes in visual responses to social interactions
}

\author{
Jon Walbrin *, Ioana Mihai, Julia Landsiedel, Kami Koldewyn \\ School of Psychology, Bangor University, Wales, United Kingdom
}

\section{A R T I C L E I N F O}

\section{Keywords:}

Social interaction

fMRI

Development

pSTS

Category selectivity

\begin{abstract}
A B S T R A C T
Recent evidence demonstrates that a region of the posterior superior temporal sulcus (pSTS) is selective to visually observed social interactions in adults. In contrast, little is known about neural responses to social interactions in children. Here, we used fMRI to ask whether the pSTS is 'tuned' to social interactions in children at all, and if so, how selectivity might differ from adults. This was investigated in the pSTS, along with several other socially-tuned regions in neighbouring temporal cortex: extrastriate body area, face selective STS, fusiform face area, and mentalizing selective temporo-parietal junction.

Both children and adults showed selectivity to social interaction within right pSTS, while only adults showed selectivity on the left. Adults also showed both more focal and greater selectivity than children (6-12 years) bilaterally. Exploratory sub-group analyses showed that younger children (6-8), but not older children (9-12), are less selective than adults on the right, while there was a continuous developmental trend (adults $>$ older $>$ younger) in left pSTS. These results suggest that, over development, the neural response to social interactions is characterized by increasingly more selective, focal, and bilateral pSTS responses, a process that likely continues into adolescence.
\end{abstract}

\section{Introduction}

As a deeply social species, humans attend to, and draw social inferences from, a wide array of social cues from both individuals and the interactions that take place between them. Social interactions often carry unique social information (e.g. shared or opposing intentions, cues to relationships, or cues to the relative social status of interactors; Quadflieg and Koldewyn, 2017). The importance of these cues is emphasized by evidence that young children are sensitive to interactive cues and can use aspects of interactive behaviour to make their own social choices. For example, infants $(<18$ months) are sensitive to the collaborative intent of an interacting dyad (Fawcett and Gredebäck, 2013; Henderson and Woodward, 2011) and show a preference for interactors who help others (Hamlin et al., 2007). However, processing other types of interactive information, such as recognising the relative social status of two interactors (Brey and Shutts, 2015), inferring the presence of an unseen interaction partner from video footage of an individual (Balas et al., 2012), and interpreting the communicative intent of interacting point-light figures (Centelles et al., 2013), undergoes substantial development across childhood.

Recent behavioural work exploring responses to human dyads in adults (e.g. Papeo et al., 2017; Ding et al., 2017; Vestner et al., 2019;
Papeo and Abassi, 2019; Papeo and Soto-Faraco, 2019) suggests that dyads that appear to be interacting are processed more similarly to one entity than two individuals. In addition, the presence of an interactant can bias the perception of their interaction partner's emotional expression (Gray et al., 2017), while noise-masked human point-light interactants are better detected in displays where their partners are performing meaningful communicative gestures (Manera et al., 2011). Similarly, point-light human dyads are better recognized in noise when their movements are synchronised (Neri et al., 2006). This evidence, in turn, suggests that the brain may process interactions differently than as a simple combination of multiple bodies, faces, and actions.

Functional magnetic resonance imaging (fMRI) evidence has begun to elucidate the neural basis of perceiving social interactions in adults. Recent work suggests specialised processing for dynamic interactions in the posterior superior temporal sulcus (pSTS) - especially in the right hemisphere - for both point-light figures and moving geometric shapes (i.e. Isik et al., 2017; Walbrin et al., 2018). These findings suggest that the pSTS not only demonstrates selectivity to social interactions (i.e. response to two interacting individuals $>$ two non-interacting individuals), but also differentiates between different types of interactions (i.e. competition and cooperation). Indeed, the pSTS is sensitive to a wide variety of visually presented dynamic human interactions

\footnotetext{
* Corresponding author.

E-mail address: jon.walbrin@gmail.com (J. Walbrin).
} 
(Centelles et al., 2011; Georgescu et al., 2014; Lahnakoski et al., 2012), interactions depicted by moving geometric shapes (e.g. Castelli et al., 2000), as well as static interaction stimuli (for a review see Quadflieg et al., 2015; Kujala et al., 2012). These results emphasize the important role that this region plays in perceiving and understanding social interactions, but it is also clear that activity in this region does not tell the whole story. Indeed, another line of recent research implicates the complementary functioning of neighbouring extrastriate body area (EBA) in the configural processing of both dynamic (Walbrin and Koldewyn, 2019) and static interacting human dyads (Abassi and Papeo, 2020). Additionally, responses across posterior-temporal regions, including EBA and pSTS, are sensitive to the apparent congruency of an interacting dyad (i.e. whether or not the two interactants' poses and appearances form a coherent interaction; Quadflieg et al., 2015). These findings suggest the important role that posterior temporal cortex plays in the visual perception of interactions.

An important extension of this work is to study how these functionally specific responses, especially in the pSTS, emerge and change during development. Previous developmental studies investigating functional pSTS responses to social stimuli have typically focused on faces and bodies, and tend to show weaker responses in children compared to adults (Deen et al., 2017; Ross et al., 2014; Scherf et al., 2007), although this is not always the case (e.g. Golarai et al., 2007). Indeed, the broader STS area is known to undergo substantial structural changes between childhood and adulthood (Bonte et al., 2013; Gogtay et al., 2004; Mills et al., 2012). Therefore, developmental changes in pSTS responses to social interactions seem likely.

To our knowledge, there has been only one developmental study looking at fMRI responses to social interactions. Sapey-Triomphe et al. (2017) compared whole-brain responses to interacting point-light figure dyads with similar, but non-interacting dyads in adults (20+ years), adolescents (13-17 years) and children (8-11 years). Although the pSTS was activated across all three age-groups, no activation differences were shown within the pSTS. Instead, parametric analyses showed a tendency for greater recruitment of fronto-parietal regions, and conversely, lesser recruitment of temporo-occipital regions with increasing age. Importantly, subjects made a social judgement for each trial (i.e. identifying if individuals were 'acting together or separately'), and as such, the resulting activation reflects not only visual processing but also processing involved in making explicit social inferences.

The present work addresses a similar question to Sapey-Triomphe et al. (2017), but with two crucial methodological differences. First, to allow greater sensitivity to potential differences between groups in the pSTS and other 'social brain' regions, we adopt a functionally defined region of interest (ROI) approach for our primary analyses. One advantage of this approach over group-level whole-brain analysis is that it is more robust to inter-subject spatial variability in activation maps (Saxe et al., 2006), an important consideration given previously observed age-related increases in the inter-subject variability of functional responses and morphological changes in superior temporal cortex (Bonte et al., 2013). Second, we attempted to minimize the contribution of top-down inferential processing, asking participants to simply watch the interaction stimuli, rather than to make explicit social judgements.

In the present study, we asked if pre-adolescent children (aged 6-12 years) show interaction selective responses (i.e. interaction $>$ noninteraction) in the pSTS (pSTS-I), and if so, whether selectivity would differ from that of adults. We also explored responses in 4 other sociallytuned posterior temporal regions that might also plausibly show agegroup differences in response to interactive stimuli: EBA, face selective STS (STS-F), fusiform face area (FFA), and mentalizing-selective temporo-parietal junction (TPJ-M). Finally, we ran exploratory analyses to determine if there was evidence for developmental change across the age range we tested, as evinced by differences between sub-groups of younger children (6-8 year olds), older children (9-11 year olds), and adults.

\section{Methods}

\subsection{Participants}

31 children aged between $6-12$ years (mean age $=8.94 ; \mathrm{SD}=1.88$; 13 females) took part in the experiment, along with 29 adults (mean age $=23.14$ years; $\mathrm{SD}=4.21$; range $=18-35 ; 16$ females). All subjects were right hand dominant. Children gave informed assent (consent was also given by a guardian of each child) and they received gift vouchers (or toys of equivalent value) as compensation for participation. Adult subjects gave consent and received monetary compensation for participation. Ethical procedures were approved by the Bangor University psychology ethics board.

\subsection{MRI tasks \& experimental session}

All scans were acquired in one session that was split into two halves with a short break where subjects came out of the scanner for approximately 5-10 minutes. This served to minimize fatigue in children, but for consistency, adults also took this break. Additionally, children also completed a head-motion 'training-session' prior to entering the scanner (see Supplementary section A). Inside the scanner, two different video tasks were used to localize and test responses within brain regions that are sensitive to: 1) dynamic social interactions; and 2) dynamic faces and bodies. For both of these tasks, subjects were not instructed to make explicit judgements about the stimuli, but instead to simply to watch the videos.

The dynamic social interaction task was almost identical to that used previously (Isik et al., 2017; Walbrin et al., 2018; see Fig. 1 for example stimuli) and consisted of three runs of videos from three conditions: Interaction (i.e. two profile-view human point-light figures interacting with each other), non-interaction (i.e. two profile-view human point-light figures performing non-interactive actions separated by a vertical line, for example, one figure jumping, the other cycling), and scrambled interaction (i.e. average 'motion-matched' scrambled versions of the interactive stimuli where the coordinates of each point-light dot were randomly shifted to disrupt the perception of interactive or biological motion) (block length $=16 \mathrm{~s}$, based on three videos of variable length that summed to $16 \mathrm{~s} ; 3 \times 16 \mathrm{~s}$ rest blocks, one presented at the beginning, middle, and end of each run; total run length $=144 \mathrm{~s}$ ). Each run consisted of two blocks per condition - one presented in either half of each run - in counterbalanced order with the other conditions (see Supplementary Table SD1).

Interaction selective pSTS-I ROIs were localized with the interaction

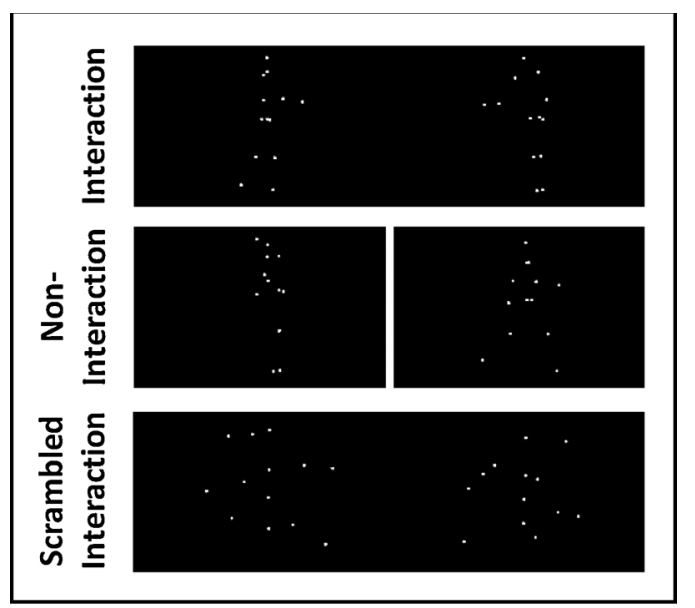

Fig. 1. Example stimuli from the social interaction task. Each panel depicts a single video frame containing two point-light figures (or scrambled point-light figures in the bottom panel) for each of the 3 conditions. 
$>$ scrambled interaction contrast. This not only captured differences in interactive content, but also biological motion (unlike the more 'closely matched' interaction $>$ non-interaction contrast that does not capture large differences in biological motion). This 'broader' contrast was chosen as it was more comparable to other localizer contrasts that were used here, and are typically used elsewhere (e.g. Julian et al., 2012; faces $>$ objects, rather than the relatively more 'socially matched' faces $>$ bodies), and to account for the possibility that weaker interaction responses in children may have resulted in poorer localization of pSTS-I ROIs.

The face and body task was adapted from a proctocol used previously (Pitcher et al., 2011), and served to localize face selective STS-F and FFA, along with body selective EBA. Participants completed three runs that contained blocks of videos that depicted either moving faces, moving bodies, or moving objects (STS-F \& FFA localization contrast = faces $>$ objects; EBA localization contrast $=$ bodies $>$ objects; block length $=18 \mathrm{~s}(6 \times 3 \mathrm{~s}$ videos $) ; 4$ blocks per condition; $5 \times 16 \mathrm{~s}$ rest blocks; total run length $=296$ s; see Supplementary Table SD2 for block counterbalancing scheme).

Additionally, mentalizing selective temporo-parietal cortex (TPJ-M) was localized using the Pixar short-film 'Partly Cloudy' (2009), by modelling responses to timepoints that reliably evoke responses to mentalizing (along with 'pain', 'social' and 'control' time-points) as described previously (Richardson et al., 2018). However, as this region did not respond strongly to interaction stimuli, these data are not reported in the main text (see Supplementary section C).

\subsection{MRI parameters, pre-processing, \& GLM estimation}

Scanning was performed with a Philips $3 \mathrm{~T}$ scanner at Bangor University. The same fMRI parameters were used for all tasks as follows: T2*-weighted gradient-echo single-shot EPI pulse sequence (with SofTone noise reduction); $\mathrm{TR}=2000 \mathrm{~ms}, \mathrm{TE}=30 \mathrm{~ms}$, flip angle $=83^{\circ}$, FOV $(\mathrm{mm})=240 \times 240 \times 112$, acquisition matrix $=80 \times 78$ (reconstruction matrix $=80$ ); 32 contiguous axial slices in ascending order, acquired voxel size $(\mathrm{mm})=3 \times 3 \times 3.5$ (reconstructed voxel size $=3 \mathrm{~mm}^{3}$ ). Four dummy scans were discarded prior to image acquisition for each run. Structural images were obtained with the following parameters: T1weighted image acquisition using a gradient echo, multi-shot turbo field echo pulse sequence, with a five echo average; $T R=12 \mathrm{~ms}$, average $\mathrm{TE}=3.4 \mathrm{~ms}$, in $1.7 \mathrm{~ms}$ steps, total acquisition time $=136 \mathrm{~s}, \mathrm{FA}=8^{\circ}, \mathrm{FOV}$ $=240 \times 240$, acquisition matrix $=240 \times 224$ (reconstruction matrix $=$ $240) ; 128$ contiguous axial slices, acquired voxel size $(\mathrm{mm})=1.0 \times 1.07$ $\times 2.0$ (reconstructed voxel size $=1 \mathrm{~mm}^{3}$ ). Pre-processing (i.e. realignment \& re-slicing, co-registration, segmentation, normalization, and smoothing) and general linear model (GLM) estimation were performed with SPM12 (fil.ion.ucl.ac.uk/spm/software/spm12). All SPM12 default pre-processing parameters were used except for the use of a $6 \mathrm{~mm}$ FWHM Gaussian smoothing kernel, and all analyses were performed in normalised MNI space with $2 \mathrm{~mm}$ isotropic voxels. Block durations and onsets for each experimental condition (per run) were modelled by convolving the corresponding box-car time-course with a canonical hemodynamic response function (without time or dispersion derivatives), with a high-pass filter of $128 \mathrm{~s}$ and autoregressive AR(1) model. Head motion parameters ( 3 translation $\& 3$ rotation axes) were modelled as nuisance regressors.

\subsection{ROI definition \& percent signal change extraction}

A group-constrained ROI definition procedure (see Julian et al. (2012) for a detailed overview of this approach) was implemented as follows. Firstly, for each given localization contrast (e.g. interaction $>$ scrambled interaction), a subject-specific ROI 'search sphere' was created by using all other within-group subjects' data to run a whole-brain analysis (see Fig. 2 for representative subject search spheres and MNI coordinates). This was used to find the voxel with the highest t-value for

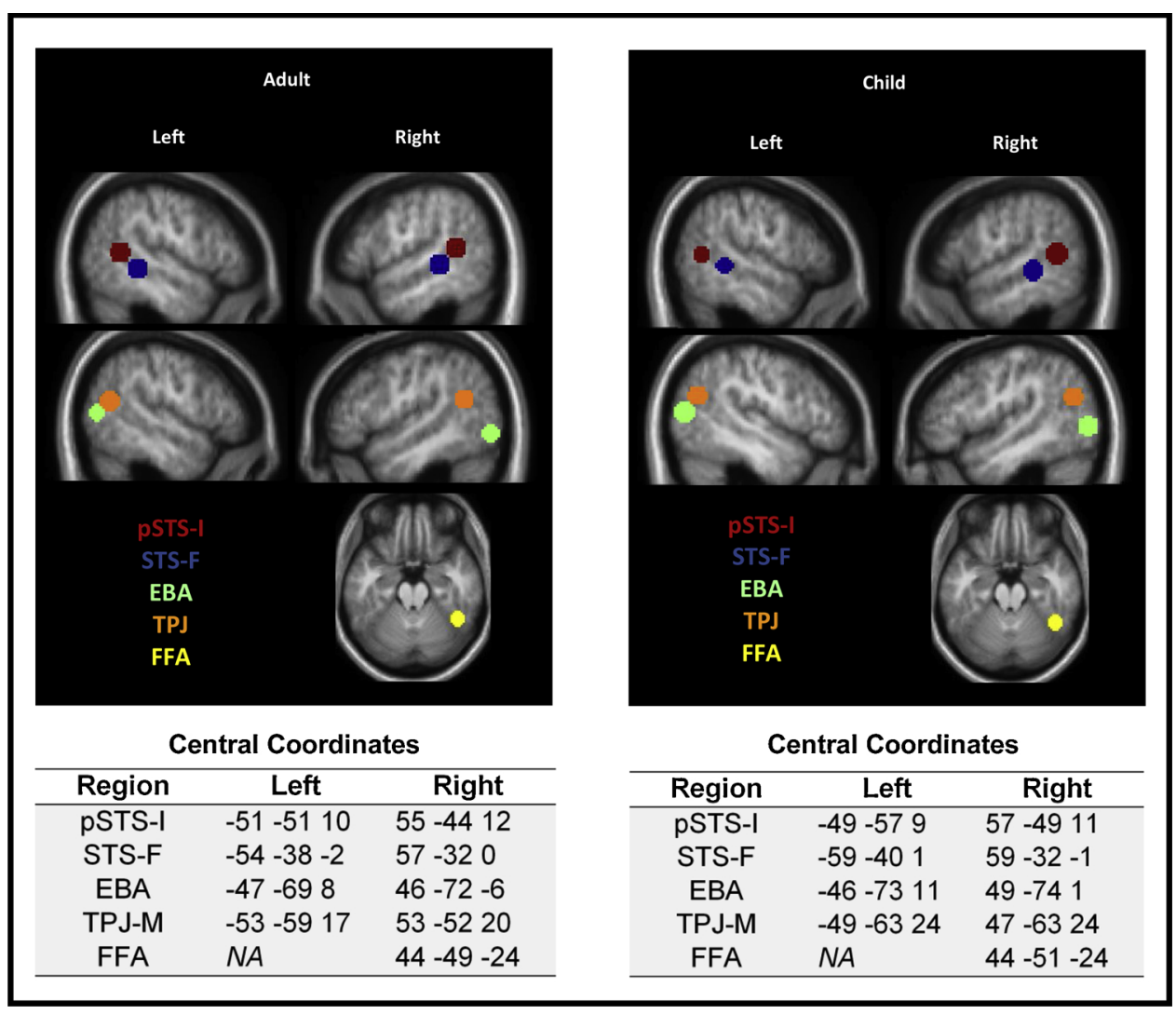

Fig. 2. Example ROI search spheres for both an example adult (left panel) and child (right panel). Each sphere is color coded with the labels in the bottom left corner of each panel. Coordinates (MNI) for the central voxel in each search space are presented for each group, respectively. pSTS-I = posterior superior temporal sulcus (interactions); STS-F $=$ superior temporal sulcus (faces); EBA = extrastriate body area; TPJ-M = temporoparietal junction (mentalizing); FFA = fusiform face area. Note: Subject-specific search spheres were generated with a leave-one-subject-out approach, and therefore the reported coordinates are representative of other subjects within each respective group (i.e. search spheres did not differ substantially across other subjects within each respective group). 

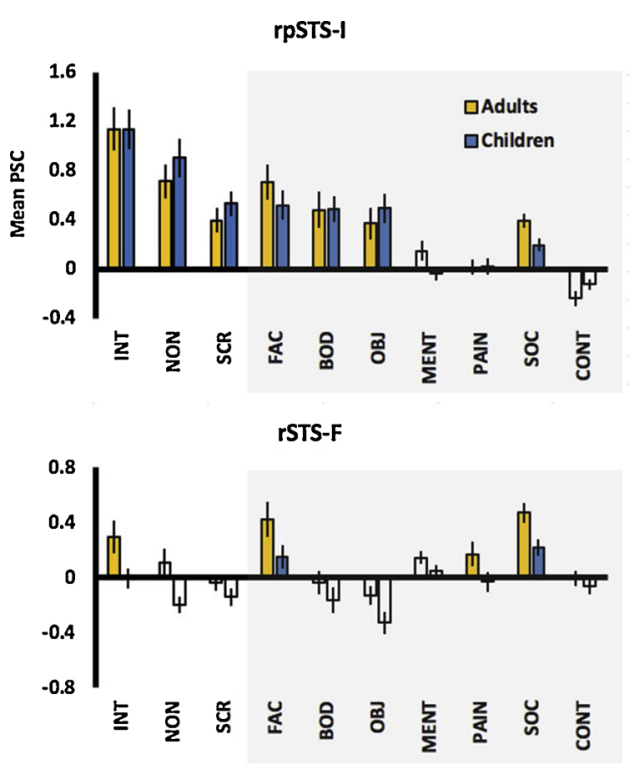
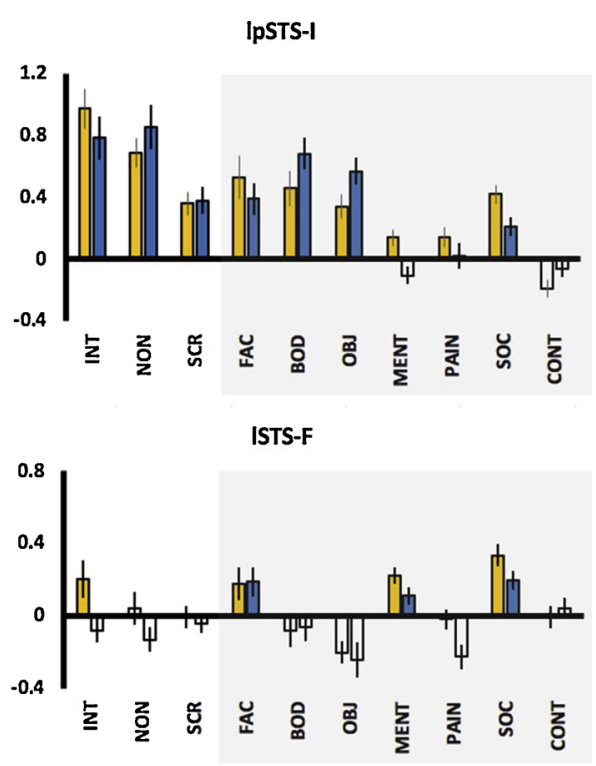

Fig. 3. Mean PSC for the 3 interaction task conditions (unshaded region) along with other conditions (shaded region) in the right and left pSTS-I ROIs. Bilateral STS-F ROIs are also shown for comparison. White bars correspond to PSC values that were not significantly greater than zero (i.e. one-sample $p$ value $>.05$ ). Error bars are SEM. INT = interaction; NON = noninteraction; SCR = scrambled interaction; FAC = faces; $\mathrm{BOD}=$ bodies; OBJ = objects; MENT = mentalizing; PAIN = pain; SOC $=$ social; CONT $=$ control. pSTS-I $=$ posterior superior temporal sulcus (interactions); STS-F = superior temporal sulcus (faces). the corresponding localization contrast where an 8mm-radius search sphere was then placed. For example, all adult group data, minus that individual, were used to create the search sphere for a given adult. This sphere size was chosen to ensure no overlap between search spheres for nearby but distinct regions (e.g. pSTS-I and STS-F) so that each ROI was comprised of unique voxels.

Subject-specific search spaces were then used to create the final set of ROIs for each participant, comprised of the 100 most activated contiguous voxels for the same contrast as that used to define the corresponding search space. The rationale for using 100 voxels was based on a compromise between using a much smaller number of voxels (e.g. 20 voxels) that might have resulted in exaggeratedly high selectivity values, and the maximum size of voxels within the search space $(200+$ voxels). However, 9 other similar sets of ROIs based on the highest 20, $40,60,80,120,140,160,180$ and 200 voxels were also generated for replication and exploratory analyses.

For the interaction task as well as face and body task (for which there were 3 runs of data), a leave-one-run-out (LORO) approach was used to ensure that data used to define ROIs was independent of that used to extract percent signal change (PSC) values. Subject-wise PSC extraction for each condition within each left-out run was performed in MarsBaR (Brett et al., 2002) and the resulting values were averaged across all LORO iterations.

\section{5. $P S C \&$ selectivity analyses}

Mean PSC was extracted for each subject, for each task, yielding a total of 10 conditions: 3 interaction task conditions (interaction, noninteraction, \& scrambled interaction) +3 face and body task conditions (faces, bodies, \& objects) +4 mentalizing task conditions (mentalizing, pain, social, \& control). PSC values for each subject were extracted from all 9 ROIs (4 bilateral ROIs and right FFA; left FFA could not be localized due to very weak responses across subjects) and then entered into group-analyses; a series of one-sample $t$-tests were performed to determine which conditions activated each ROI above baseline. Above-zero PSC values for a given 'target condition' (i.e., interaction, faces, bodies, mentalizing) were considered a pre-requisite for calculating selectivity scores, as any region not univariately driven by a given target condition cannot be interpreted as meaningfully responsive to that given category. Therefore, selectivity scores were not calculated for contrasts in regions that did not show above-zero PSC responses for given target conditions.
Interaction selectivity was calculated for each subject, for each ROI, with the following PSC subtraction: Interaction - non-interaction. For comparison analyses, face and body selectivity measures were also calculated as faces - objects, and bodies - objects, respectively. Subjects' selectivity values were then entered into group analyses (i.e. ANOVAs \& $t$-tests) for each ROI.

Finally, Bonferroni multiple-comparison correction was implemented for each 'set' of analyses separately (but not for the exploratory analyses in Sections $3.6 \&$ 3.7). The corrected Bonferroni threshold $(\alpha)$ is stated for each series of tests in the results section. We focus on results that survive Bonferroni correction, but in keeping with the continuousnature of p-values, we also report results that are significant at an uncorrected level (i.e. $\mathrm{p}<.05)$, along with marginal trends $(\mathrm{p}<.10)$. Importantly, we only make strong claims for results that survive Bonferroni correction, and explicitly state uncorrected and marginal findings. All one-sample $t$-test p-values are one-tailed.

\subsection{Controlling for head motion}

In line with previous developmental studies (e.g. Peelen et al., 2009), we excluded runs of data with $>2 \mathrm{~mm}$ scan-to-scan movement. This resulted in the exclusion of single runs of data in three separate children (although including these runs did not meaningfully change any results). In addition to removing these runs, differences in head motion between groups were tested using an analysis similar to Kang et al. (2003). Crucially, these results revealed that any age-group differences in selectivity are not attributable to head motion differences (see Supplementary section $\mathrm{B}$ ).

\section{Results}

\subsection{PSC responses}

Before calculating selectivity values, we inspected PSC for each condition, across tasks. Stronger responses to the interaction condition than face, body, or mentalizing conditions were shown in bilateral pSTSI ROIs for adults. The same pattern was observed for children in right pSTS-I, while in the left pSTS-I, the response to the non-interaction condition was slightly greater than for the interaction condition. Additionally, no other region differentiated between the interaction and noninteraction conditions except for bilateral STS-F ROIs in adults (see Fig. 1; see Supplementary figure SE1 for PSC charts for other ROIs). 


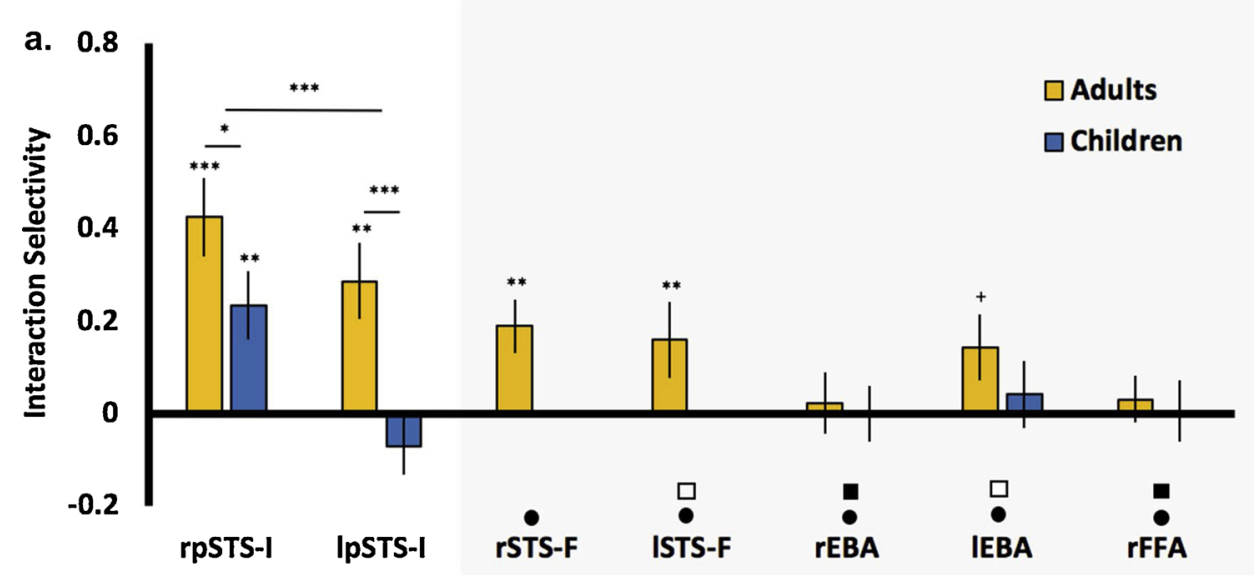

b.

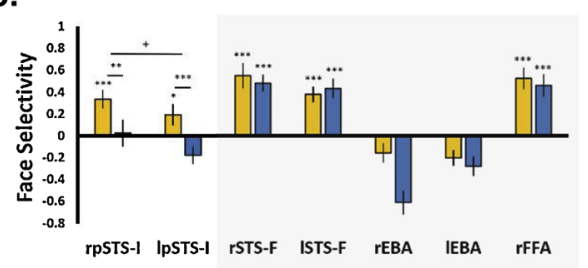

Fig. 4. (a) Mean interaction selectivity values for the pSTS-I ROIs (unshaded region) and other ROIs (shaded region). ${ }^{* * *}=p<.001 ;{ }^{* *}=p<$ $.01 ; *=<.05 ;{ }^{+} p<.057$. Error bars are SEM. Black circles denote regions that are significantly less selective than right pSTS-I for both age-groups. Black and white squares, respectively, denote regions that are either significantly or marginally less selective than the left pSTS-I in adults. (b) Mean face selectivity and (c) mean body selectivity across ROIs are shown for comparison. pSTS-I = posterior superior temporal sulcus (interactions); STS-F = superior temporal sulcus (faces); EBA = extrastriate body area; FFA = fusiform face area.
These findings also show that PSC responses in children are not uniformly weaker than adults; indeed, children showed approximately equal or greater PSC values than adults for several conditions across ROIs. Therefore, any differences in selectivity are not attributable to weaker overall PSC responses in children.

\subsection{Interaction selectivity in $p S T S-I$}

A series of analyses were performed to test interaction selectivity in each ROI (see Fig. 4). Regions for which selectivity was not calculated (i. e. due to PSC values that were not above zero) are shown in Fig. 3 (see white bars). One-sample $t$-tests (uncorrected) were then performed to determine which regions showed above-zero selectivity for interactions (see Supplementary Table SF1 for one-sample statistics for interaction selectivity values). For adults, strong interaction selectivity was shown for the right $(p<.001)$ and left pSTS-I $(p=.002)$, and bilateral STS-F (both $p s<.005)$, and marginal selectivity in the left EBA $(p=.054)$. By contrast, the right pSTS-I was the only interaction selective region in children $(p=.002)$.

Next, interaction selectivity in right pSTS-I and left pSTS-I was compared with a $2 \times 2$ mixed ANOVA (hemisphere $\mathrm{x}$ age-group). Greater responses were observed in the right pSTS-I $(F(1,58)=21.70$, $p<.001)$, and greater selectivity overall was shown for adults $(F(1,58)=$ $8.05, p=.006$ ), along with a marginal interaction, suggesting a trend for greater selectivity in adults compared to children $(F(1,58)=3.11, p=$ .083). Two follow-up $t$-tests were performed (Bonferroni corrected $\alpha=$ .025) on selectivity values in right and left pSTS-I, separately. Adults were significantly more selective than children in the left hemisphere $(t$ $(58)=3.50, p=.001)$, and in the right hemisphere $(t(58)=1.68, p=$ .049). but the contrast on the right did not survive multiple comparison correction. These findings show greater interaction selectivity for adults than children in the left pSTS-I, as supported by a large effect size (Cohen's $d=0.90$ ), with a smaller difference in the right pSTS-I, as supported by a small-to-medium effect size (Cohen's $d=0.44$ ). Thus, both adults and children show selective responses in the right pSTS-I, but unlike adults, children do not show interaction selectivity in the left pSTS-I.

\subsection{Interaction selectivity in pSTS-I vs. other ROIs}

A series of analyses were then performed to assess whether interaction selectivity was significantly greater in the pSTS-I than other ROIs (see Fig. 4; see Supplementary Table SG1 for full statistics). These results showed that for both adults and children alike, selectivity was significantly greater in the right PSTS-I (Bonferroni corrected $\alpha=.006$ ) than right EBA, left EBA, \& right FFA (all $p s \leq .002$ ). As children did not show selectivity in bilateral STS-F ROIs, comparisons for these areas were only run in adults, revealing greater selectivity for right pSTS-I for both comparisons (against right STS-F: $p=.002$; against left STS-F: $p=.008$, significant at uncorrected level only). For the left pSTS-I, only adults showed above-zero interaction selectivity, and so analyses were only performed with adult data (Bonferroni corrected $\alpha=.01$ ). Selectivity was greater in left pSTS-I than right FFA $(p=.001)$ and right EBA ( $p=$ $.003)$ at corrected levels, as well as in left STS-F $(p=.044)$ and left EBA $(p=.027)$ at uncorrected levels. However, while the response was higher in left pSTS-I than right STS-F, this difference was not significant, even at an uncorrected level ( $p=.113)$. These findings show a strong trend for greater responses in right pSTS-I compared to other ROIs, and a similar, though weaker, pattern in the left pSTS-I.

\subsection{Interaction $v$ s. face \& body selectivity}

Interaction selectivity is greater in the pSTS-I ROIs than virtually all other ROIs, but is the PSTS-I more selective for interactions than for faces or bodies? To test this, a $3 \times 2$ mixed ANOVA (selectivity category $\mathrm{x}$ age-group) with follow-up tests was performed for the right pSTS-I, along with $t$-tests in adults for the left pSTS-I (Bonferroni corrected $\alpha$ $=.01)$. In the right pSTS-I, a main effect of selectivity category ( $F$ $(1.46,80.45)=8.11, p=.001)$, and a main effect of age-group at an uncorrected level was shown $(F(1,55)=4.92, p=.031)$. As the interaction term was not significant $(F(1.46,80.45)=1.05, p=.337)$, followup paired $t$-tests were performed on both adults and children's selectivity scores together. Interaction selectivity was significantly greater than body selectivity $(t(56)=4.17, p<.001)$, and greater than face selectivity at an uncorrected threshold $(t(56)=1.75, p=.043)$.

For the left pSTS-I, adults showed greater interaction selectivity than body selectivity at an uncorrected threshold $(t(27)=2.12, p<.022)$, 
and the trend for greater interaction than face selectivity was not significant $(t(27)=1.12, p=.132)$. These results demonstrate that, for both adults and children alike, interaction selectivity in the right pSTS-I is greater than body selectivity, and to a lesser extent, greater than face selectivity. These effects are considerably weaker for adults in the left pSTS-I (and absent in children).

\subsection{Face and body selectivity in pSTS-I}

We also compared face and body selective responses in pSTS-I (along with responses in STS-F, and EBA, respectively; see Fig. 4(b) and Supplementary sections $\mathrm{F} \& \mathrm{H}$ for full statistics). A $2 \times 2$ ANOVA (hemisphere $x$ age-group) revealed that face selectivity was marginally greater in right than left pSTS-I $(p=.057)$, and adults were more selective than children $(p=.002)$ with no interaction between factors $(p=.737)$. By contrast, comparing face selectivity in right and left STS-F ROIs, for both groups, revealed no significant ANOVA term (all $p s>.141$ ). For body selectivity, similar ANOVAs revealed no significant term for pSTS-I (all $p s>.228$ ) or EBA (all $p s>$.539). In summary, the pattern of face selectivity in bilateral pSTS-I bears some resemblance with the pattern observed for interaction selectivity, while no differences in body selectivity were shown.

\subsection{Whole-brain analyses}

To determine whether other regions outside of the functionally localized ROIs demonstrated sensitivity for the interaction > noninteraction contrast, whole-brain analyses were performed for adults and children (see Fig. 5). Individual subject t-maps for this contrast were entered into a group analysis, with the resulting group-level t-maps height-thresholded at $p=.001$, and false discovery rate (FDR) clustercorrected at $p<.05$. This group-level t-map was then projected onto an MNI template surface brain in SPM12 for visualisation.

For adults, right hemisphere responses were shown with peak activation in the pSTS, along with activations extending to the anterior STS (aSTS), and a small cluster in IFG. Additionally, bilateral precuneus and a small left calcarine sulcus activation was shown. For children, similar although weaker responses were shown in the right pSTS and aSTS regions only. When comparing activation between the two groups directly, no differences were observed for either the adults $>$ children, or the reverse contrast, demonstrating that these analyses were not as sensitive as ROI analyses at capturing group differences.

\subsection{Exploratory analysis: pSTS-I interaction selectivity as a function of ROI size}

The preceding analyses used selectivity measures that were generated from ROIs with a fixed size of 100 voxels, therefore ensuring no group differences in ROI size. We also conducted exploratory analyses to determine if selectivity differed as a function of ROI size, and if so, whether such changes were similar between groups. Interaction selectivity as a function of ROI size is shown in Fig. 6, where a clear trend for greater selectivity in adults than children is shown across all ROI sizes.

Interestingly, adults show an approximately linear decrease in selectivity with increasing ROI size, whereas this does not appear to be the case for children. To formally test this apparent trend, linear regression slopes were calculated for each subject; that is, a beta coefficient that describes the change in selectivity across ROI sizes was calculated, per ROI, per subject, and entered into a series of (uncorrected) group tests. A $2 \times 2$ mixed ANOVA (hemisphere $\mathrm{x}$ age-group) revealed that beta coefficients were significantly more negative for adults than children in bilateral pSTS-I (main effect of age-group: $F$ $(1,58)=7.05, p=.010$; non-significant main effect of hemisphere: $F$ $(1,58)=0.01, p=.912$; non-significant interaction term: $F(1,58)=0.00$, $p=.992)$; these findings show that interaction selectivity decreases linearly with increasing ROI size in adults, but not children (see Fig. 6). We speculate that this might indirectly reflect age-related differences in the focal tuning of interaction selective responses in the pSTS; that is, adults show strongest selectivity around a small peak cluster in the pSTS with intermediate selectivity in neighbouring STS cortex, while children are less selective overall and do not show graded changes in selectivity in the right pSTS (and are not selective at all in the left pSTS).

\subsection{Exploratory sub-group analyses: interaction selectivity}

The current study was designed to compare differences between adults and pre-adolescent children (aged 6-12 years), but we are also interested in whether there is developmental change across age within the child group. To test if this might be true, we compared interaction selective responses in pSTS-I across smaller sub-groups: $6-8$ years $(\mathrm{N}=$ $14), 9-11$ years $(\mathrm{N}=14)$, and a random subset of adults $(\mathrm{N}=14)$. Despite the relatively small group sizes, this exploratory analysis was intended to see whether nuanced sub-group differences in interaction selectivity might exist.

Sub-group differences in interaction selectivity were observed in the pSTS-I for the 100 voxel ROIs (see Fig. 7 (pink shaded region); see Supplementary sections I \& J for ROI regression slope and whole-brain analyses). A one-way ANOVA for the right pSTS-I revealed a marginal difference across the three sub-groups $(F(2,39)=2.53, p=.093)$; followup $t$-tests revealed that adults were more selective than younger $(t(26)=$ $1.89, p=.035)$ but not older children $(t(26)=0.49, p=.961)$, while older children were more selective than younger children $(t(26)=1.78$, $p=.044)$. Sub-group differences in the lpSTS-I $(F(2,39)=5.90, p=$ .006) were underscored by greater selectivity for adults compared to both younger $(t(26)=3.41, p=.001)$ and older children $(t(26)=1.88, p$ $=.035$ ) while a marginal trend for greater selectivity for older than

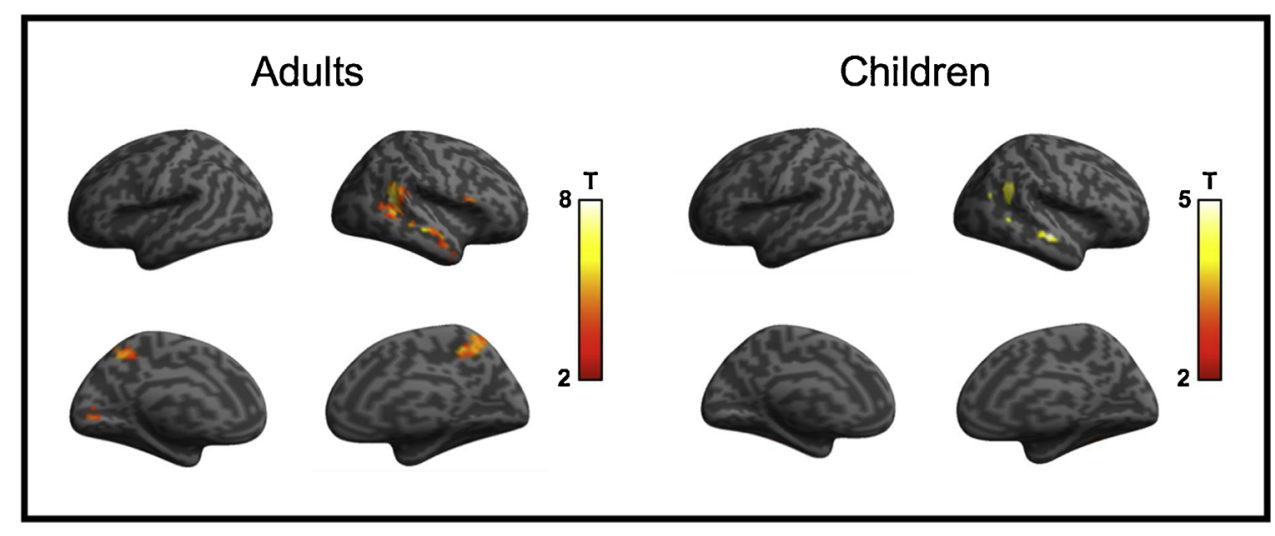

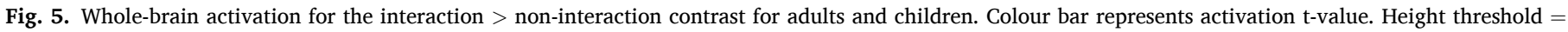
.001 ; FDR cluster correction $=.05$. 
rpSTS-I

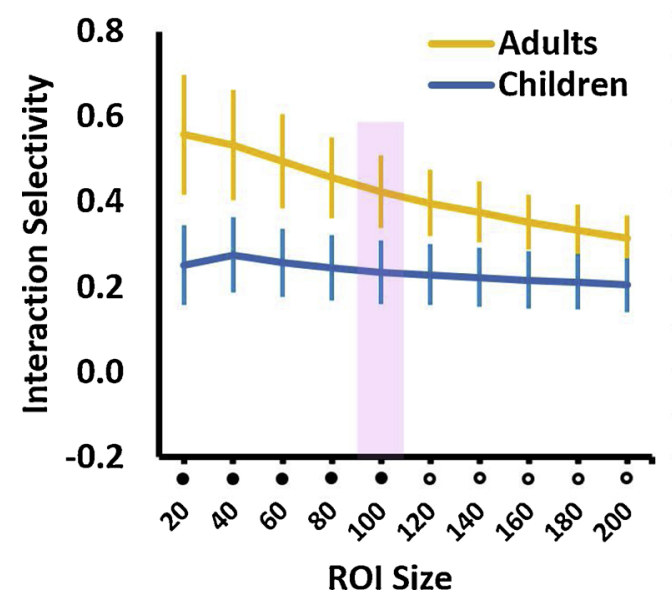

IpSTS-I

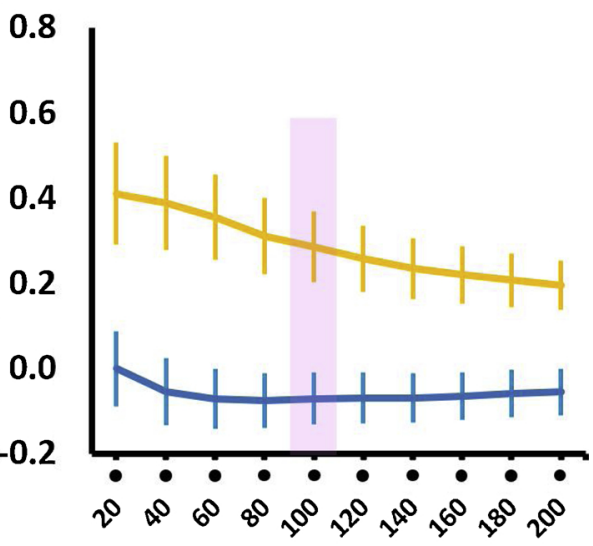

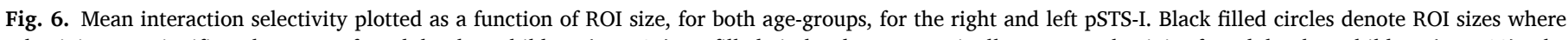

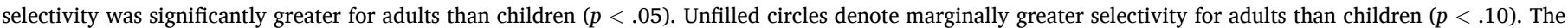
shaded region shows the 100 voxel ROI, as used in the preceding analyses. Error bars are SEM. pSTS-I = posterior superior temporal sulcus (interactions).

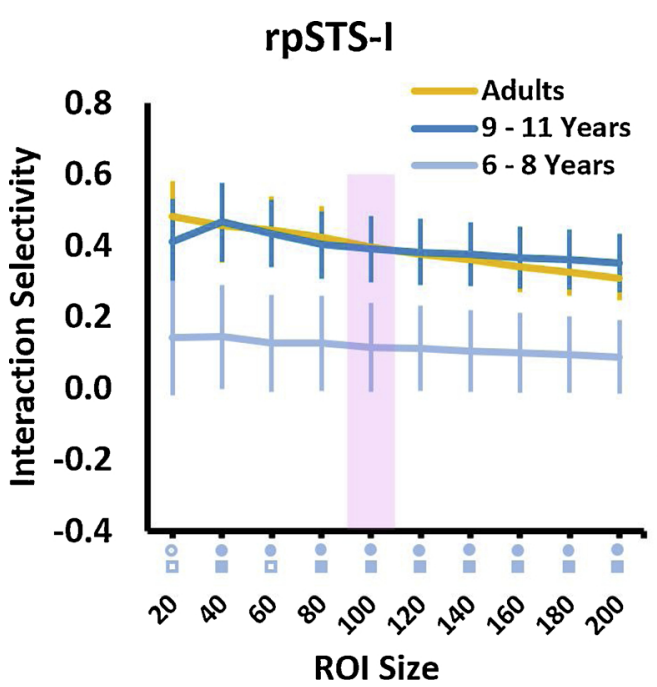

IpSTS-I

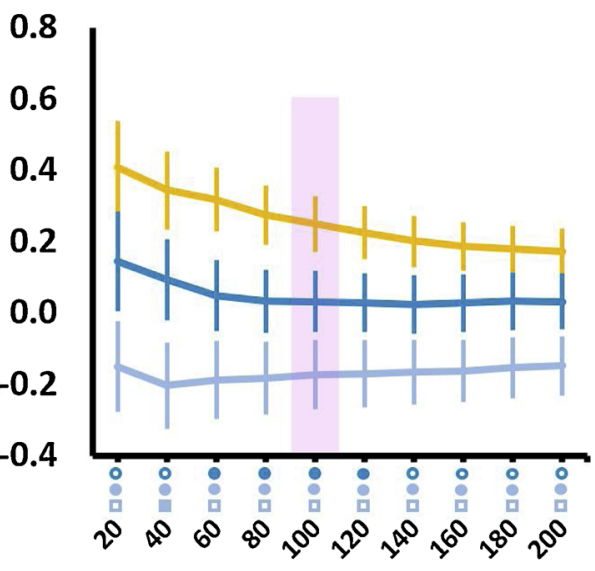

Fig. 7. Mean interaction selectivity plotted as a function of ROI size, for the three sub-groups for the right and left pSTS-I. The shaded region denotes the original 100 voxel ROI. For each ROI size, statistical effects for each sub-group $t$ test comparison are indicated by coloured symbols underneath the x-axis, as follows; dark blue circles: adults $>9-11$ years; light blue circles: adults $>6-8$ years; light blue squares: $9-11$ years $>6-8$ years; filled symbols: $p<$ .05 ; unfilled symbols: $p<.10$. Error bars are SEM. pSTS-I = posterior superior temporal sulcus (interactions). young children was observed $(t(26)=1.59, p=.063)$.

Importantly, although these results were obtained with the 100 voxel ROIs, they are robust across different ROI sizes. However, we emphasize the exploratory nature of these results, and offer only a tentative interpretation; we suggest that the observed trend in the right pSTS-I in the main interaction selectivity analysis (i.e. all adults $>$ all children) is likely driven by weaker selectivity in younger children; by contrast, both older and younger children are less selective than adults in left pSTS-I, and selectivity appears to increase with age.

\section{Discussion}

Here, we report four key findings. First, and importantly, children as young as six show selective responses to social interactions in the pSTS, implying that even in childhood, the social brain is already tuned to understand social interactions. Secondly, as predicted, adults showed greater selectivity for social interactions than children in the pSTS; this effect was strongest in the left hemisphere, where children showed no selectivity, but was only marginal in the right hemisphere, where children showed intermediate levels of interaction selectivity. Exploratory analyses suggest that the all adult $>$ all children difference in the right pSTS might be driven by weaker selectivity in the youngest children
(6-8 years), while older children (9-11 years) already demonstrated adult-like selectivity. Clear developmental trends were found in the left pSTS as a graded pattern of increasing selectivity with age across the three sub-groups (i.e. adults $>$ older children $>$ younger children). Importantly, both the main and sub-group findings generalized across different ROI sizes, suggesting that these are stable effects. Thirdly, and unexpectedly, unlike children, adults showed additional interactionspecific co-activations in other 'socially tuned' temporal lobe regions. Fourthly, adults demonstrated much more focally tuned interaction responses in bilateral pSTS, where selectivity dropped off from a core 'peak', whereas children showed weaker, broader selectivity that did not change across ROI size. Altogether, although children do already show functional selectivity for social interactions, these findings demonstrate that neural responses to social interactions are not fully mature in preadolescent children, and therefore must undergo substantial development during adolescence.

Both adults and children showed significantly greater interaction selectivity in the right pSTS than all other ROIs, and this was supported by the whole-brain findings that show this was the most active region. These findings are consistent with previous accounts that the pSTS especially in the right hemisphere - is strongly responsive to dyadic social interactions (Georgescu et al., 2014; Isik et al., 2017; Kujala et al., 
2012; Lahnakoski et al., 2012; Walbrin et al., 2018; Walbrin and Koldewyn, 2019).

Adults showed stronger interaction selective responses in the pSTS than children, especially in the left-hemisphere, in line with analogous trends for functionally localized face (Scherf et al., 2007) and body regions in the STS (Ross et al., 2014), and similarly for mentalizing responses in TPJ (Saxe et al., 2009). Additionally, adults showed strong responses in both hemispheres, and greater responses in right than left pSTS. By contrast, children demonstrated above-zero selectivity in the right but not left pSTS. Interestingly, similar developmental changes have been observed previously in the STS, albeit in a different modality. Bonte et al. (2013) calculated laterality scores for voice selectivity in the STS (i.e. the ratio between the magnitude and extent of left and right lateralized STS responses) and found that both children and adults showed rightward lateralization. Crucially, this effect was significantly stronger in children, as proportionally more recruitment of left STS was shown for adults. Additionally, voice selective responses in the STS were more diffuse and less selective in children than adults (who showed both strong and spatially constrained selectivity), mirroring age related differences in tuning of interaction selectivity in the current study. These results suggest that selective responses in the STS may become relatively more bilateral and focally tuned across development (although this trend is not pronounced for faces in our data).

It is also worth noting that 'non-selectivity' for interactions in the left pSTS in children is not the result of weaker interaction responses per se; instead, strong PSC responses were found for both interactions and noninteractions, but they were similar in magnitude. This could be interpreted in two ways. Firstly, that responses in this region are driven by the mere presence of two individuals, but not by interactive information (e.g. contingent actions and facing direction). As such, immature responses in this region may reflect simplistic representations of interactions as merely two people together, that are insensitive to nuanced dynamic information that adults make use of to distinguish these two conditions. And secondly, responses in this region may simply reflect sensitivity to biological motion per se, that was approximately equivalent between interaction and non-interaction stimuli. This is supported by previous evidence of STS sensitivity to biological motion in children (e.g. Mosconi et al., 2005; Carter and Pelphrey, 2006) and additionally, PSC responses to the body condition (from the face and body task) were comparable to interactions in the left pSTS but not right pSTS.

Further to this, adults, but not children, unexpectedly showed complementary interaction selective responses in regions neighbouring interaction selective pSTS (e.g. face selective STS cortex and to a marginal extent, left EBA). Along with strong focal tuning in the pSTS, weaker selectivity in neighbouring face selective STS is somewhat unsurprising, given the relevance of facial information in interactive contexts. Indeed, a similar pattern was shown for face information, whereby stronger face selective responses were found in face selective STS, with weaker face selectivity in neighbouring interaction selective pSTS in adults. Partially overlapping STS responses are shown for other categories of social information (e.g. theory of mind, biological motion, faces, and voices; Deen et al., 2015; Lahnakoski et al., 2012) that are likely important to understanding the content of social interactions. While previous research in adults has demonstrated pSTS-I responds selectively even when interactions do not contain any human information (i.e., when interactants are animate moving shapes; Isik et al., 2017; Walbrin et al., 2018), our data suggests that other social regions in the temporal lobe may also, by adulthood, develop at least some sensitivity to social interaction cues. While the present findings focus on dynamic whole-body dyadic interactions, further research could address the possibility that such 'interaction tuning' varies by social category along the extent of the STS (e.g. posterior STS interaction selectivity for bodies may be complemented with more anterior tuning to face- and voice-based interactions).

The whole-brain findings reported here are somewhat similar to the results of Sapey-Triomphe et al. (2017; i.e. using a highly similar stimulus set and the same interaction $>$ non-interaction contrast). In that study, the authors reported strong pSTS (and wider posterior temporal cortex) responses across adults, adolescents, and children (8-11 years), along with activation in IFG. These results are largely replicated by the present analyses; both adults and children (but not younger children in sub-group analyses) showed responses in right pSTS and aSTS. Although we did not predict responses in the aSTS, co-activation of pSTS and aSTS have been previously shown for social interactions (Lahnakoski et al., 2012), and dynamic faces (Pitcher et al., 2011), and may suggest functional coupling between these regions, although whether these regions perform similar or different computations during interaction perception remains to be seen. Unlike Sapey-Triomphe et al. (2017), we did not use an explicit judgement task, but instead asked subjects to simply watch the stimuli. While it is possible that group differences in visual attention or eye-gaze patterns might emerge under such free-viewing conditions, we did not observe fronto-parietal activations that are associated with top-down modulation of visual attention or differential eye-gaze responses (i.e. inferior parietal sulcus and frontal eye-fields; e.g. Corbetta and Shulman, 2002) in any whole-brain analysis. Similarly, unpublished pilot data from our lab shows equivalent PSC responses in pSTS with or without the vertical separation line in the non-interaction stimuli, and therefore attentional differences resulting from the presence of this line are unlikely. Likewise, PSC responses for adults and children were comparable for most conditions, in most ROIs. However, it is possible that more subtle group differences might exist, and that this certainly merits further investigation.

The present findings demonstrate that the right pSTS in preadolescent children already shows selectivity to social interactions, similar to that seen in adults. However, children also show markedly different neural responses to dyadic social interactions. These results imply that the maturation of neural interaction selectivity is characterized by increasingly bilateral pSTS activation, increasingly stronger selectivity and more focal tuning bilaterally, and increased, though nonselective, responses to social interaction in neighbouring temporal cortex. These findings should motivate further research to explore interaction responses across the full life span, including adolescence and late adulthood, and to explore developmental changes in the context of other types of interactions (e.g. stimuli that are restricted to faces or voices).

\section{Author contributions}

J.W \& K.K: study design, data collection, data analysis, manuscript writing and editing; I.M \& J.L: data analysis \& data collection.

\section{Declaration of Competing Interest}

None declared.

\section{Acknowledgement}

This work has received funding from the European Research Council under the European Union's Horizon 2020 research and innovation programme (ERC-2016-STG-716974: Becoming Social).

\section{Appendix A. Supplementary data}

Supplementary material related to this article can be found, in the online version, at doi:https://doi.org/10.1016/j.dcn.2020.100774.

\section{References}

Abassi, E., Papeo, L., 2020. The representation of two-body shapes in the human visual cortex. J. Neurosci. 40 (4), 852-863.

Balas, B., Kanwisher, N., Saxe, R., 2012. Thin-slice perception develops slowly. J. Exp. Child Psychol. 112 (2), 257-264.

Bonte, M., Frost, M.A., Rutten, S., Ley, A., Formisano, E., Goebel, R., 2013. Development from childhood to adulthood increases morphological and functional inter- 
individual variability in the right superior temporal cortex. NeuroImage 83, 739-750.

Brett, M., Anton, J.L., Valabregue, R., Poline, J.B., 2002. Region of interest analysis using an SPM toolbox. Paper Presented at 8th International Conference on Functional Mapping of the Human Brain, Sendai, Japan. Retrieved from: https://matthew. dynevor.org/research/abstracts/marsbar/marsbar_abstract.pdf.

Brey, E., Shutts, K., 2015. Children use nonverbal cues to make inferences about social power. Child Dev. 86, 276-286.

Carter, E.J., Pelphrey, K.A., 2006. School-aged children exhibit domain-specific responses to biological motion. Soc. Neurosci. 1 (3), 396-411.

Castelli, F., Happé, F., Frith, U., Frith, C., 2000. Movement and mind: a functional imaging study of perception and interpretation of complex intentional movement patterns. Neuroimage 12 (3), 314-325.

Centelles, L., Assaiante, C., Nazarian, B., Anton, J.L., Schmitz, C., 2011. Recruitment of both the mirror and the mentalizing networks when observing social interactions depicted by point-lights: a neuroimaging study. PLoS One 6, e15749.

Centelles, L., Assaiante, C., Etchegoyhen, K., Bouvard, M., Schmitz, C., 2013. From action to interaction: exploring the contribution of body motion cues to social understanding in typical development and in autism spectrum disorders. J. Autism Dev. Disord. 43 (5), 1140-1150.

Corbetta, M., Shulman, G.L., 2002. Control of goal-directed and stimulus-driven attention in the brain. Nat. Rev. Neurosci. 3 (3), 201.

Deen, B., Koldewyn, K., Kanwisher, N., Saxe, R., 2015. Functional organization of social perception and cognition in the superior temporal sulcus. Cereb. Cortex 25 (11), 4596-4609.

Deen, B., Richardson, H., Dilks, D.D., Takahashi, A., Keil, B., Wald, L.L., et al., 2017. Organization of high-level visual cortex in human infants. Nat. Commun. 8, 13995

Ding, X., Gao, Z., Shen, M., 2017. Two equals one: two human actions during social interaction are grouped as one unit in working memory. Psychol. Sci. 28 (9), 1311-1320.

Fawcett, C., Gredebäck, G., 2013. Infants use social context to bind actions into a collaborative sequence. Dev. Sci. 16 (6), 841-849.

Georgescu, A.L., Kuzmanovic, B., Santos, N.S., Tepest, R., Bente, G., Tittgemeyer, M. Vogeley, K., 2014. Perceiving nonverbal behavior: neural correlates of processing movement fluency and contingency in dyadic interactions. Hum. Brain Mapp. 35 (4), 1362-1378.

Gogtay, N., Giedd, J.N., Lusk, L., Hayashi, K.M., Greenstein, D., Vaituzis, A.C., et al., 2004. Dynamic mapping of human cortical development during childhood through early adulthood. Proc. Natl. Acad. Sci. 101 (21), 8174-8179.

Golarai, G., Ghahremani, D.G., Whitfield-Gabrieli, S., Reiss, A., Eberhardt, J.L., Gabrieli, J.D., Grill-Spector, K., 2007. Differential development of high-level visual cortex correlates with category-specific recognition memory. Nat. Neurosci. 10 (4), 512.

Gray, K.L., Barber, L., Murphy, J., Cook, R., 2017. Social interaction contexts bias the perceived expressions of interactants. Emotion 17 (4), 567.

Hamlin, J.K., Wynn, K., Bloom, P., 2007. Social evaluation by preverbal infants. Nature 450 (7169), 557.

Henderson, A.M., Woodward, A.L., 2011. "Let's work together": what do infants understand about collaborative goals? Cognition 121, 12-21.

Isik, L., Koldewyn, K., Beeler, D., Kanwisher, N., 2017. Perceiving social interactions in the posterior superior temporal sulcus. Proc. Natl. Acad. Sci. 114 (43), 9145-9152.

Julian, J.B., Fedorenko, E., Webster, J., Kanwisher, N., 2012. An algorithmic method for functionally defining regions of interest in the ventral visual pathway. NeuroImage 60 (4), 2357-2364.

Kang, H.C., Burgund, E.D., Lugar, H.M., Petersen, S.E., Schlaggar, B.L., 2003. Comparison of functional activation foci in children and adults using a common stereotactic space. NeuroImage 19 (1), 16-28.
Kujala, M.V., Carlson, S., Hari, R., 2012. Engagement of amygdala in third-person view of face-to-face interaction. Hum. Brain Mapp. 33 (8), 1753-1762.

Lahnakoski, J.M., Glerean, E., Salmi, J., Jääskeläinen, I.P., Sams, M., Hari, R., Nummenmaa, L., 2012. Naturalistic FMRI mapping reveals superior temporal sulcus as the hub for the distributed brain network for social perception. Front. Hum. Neurosci. 6, 233.

Manera, V., Becchio, C., Schouten, B., Bara, B.G., Verfaillie, K., 2011. Communicative interactions improve visual detection of biological motion. PLoS One 6 (1), e14594.

Mills, K.L., Lalonde, F., Clasen, L.S., Giedd, J.N., Blakemore, S.J., 2012. Developmental changes in the structure of the social brain in late childhood and adolescence. Soc. Cogn. Affect. Neurosci. 9, 123-131.

Mosconi, M.W., Mack, P.B., McCarthy, G., Pelphrey, K.A., 2005. Taking an "intentional stance" on eye gaze shifts: a functional neuroimaging study of social perception in children. NeuroImage 27, 247-252.

Neri, P., Luu, J.Y., Levi, D.M., 2006. Meaningful interactions can enhance visual discrimination of human agents. Nat. Neurosci. 9 (9), 1186.

Papeo, L., Abassi, E., 2019. Seeing social events: the visual specialization for dyadic human-human interactions. J. Exp. Psychol. Hum. Percept. Perform. 45 (7), 877.

Papeo, L., Soto-Faraco, S., 2019. Visual search for people among people. PsyArXiv. https://doi.org/10.31234/osf.io/fupes.

Papeo, L., Stein, T., Soto-Faraco, S., 2017. The two-body inversion effect. Psychol. Sci. 28 (3), 369-379.

Peelen, M.V., Glaser, B., Vuilleumier, P., Eliez, S., 2009. Differential development of selectivity for faces and bodies in the fusiform gyrus. Dev. Sci. 12 (6), 16-25.

Pitcher, D., Dilks, D.D., Saxe, R.R., Triantafyllou, C., Kanwisher, N., 2011. Differential selectivity for dynamic versus static information in face selective cortical regions. NeuroImage 56 (4), 2356-2363.

Quadflieg, S., Koldewyn, K., 2017. The neuroscience of people watching: how the human brain makes sense of other people's encounters. Ann. N. Y. Acad. Sci. 1396, 166-182.

Quadflieg, S., Gentile, F., Rossion, B., 2015. The neural basis of perceiving person interactions. Cortex 70, 5-20.

Richardson, H., Lisandrelli, G., Riobueno-Naylor, A., Saxe, R., 2018. Development of the social brain from age three to twelve years. Nat. Commun. 9, 1027.

Ross, P.D., de Gelder, B., Crabbe, F., Grosbras, M.H., 2014. Body selective areas in the visual cortex are less active in children than in adults. Front. Hum. Neurosci. 8, 941.

Sapey-Triomphe, L.A., Centelles, L., Roth, M., Fonlupt, P., Henaff, M.A., Schmitz, C., Assaiante, C., 2017. Deciphering human motion to discriminate social interactions: a developmental neuroimaging study. Soc. Cogn. Affect. Neurosci. 12 (2), 340-351.

Saxe, R., Brett, M., Kanwisher, N., 2006. Divide and conquer: a defense of functional localizers. NeuroImage 30 (4), 1088-1096.

Saxe, R.R., Whitfield-Gabrieli, S., Scholz, J., Pelphrey, K.A., 2009. Brain regions for perceiving and reasoning about other people in school-aged children. Child Dev. 80 (4), 1197-1209.

Scherf, K.S., Behrmann, M., Humphreys, K., Luna, B., 2007. Visual category-selectivity for faces, places and objects emerges along different developmental trajectories. Dev. Sci. 10 (4), F15-F30.

Vestner, T., Tipper, S.P., Hartley, T., Over, H., Rueschemeyer, S.A., 2019. Bound together: social binding leads to faster processing, spatial distortion, and enhanced memory of interacting partners. J. Exp. Psychol. Gen. 18 (5), 1251-1268.

Walbrin, J., Koldewyn, K., 2019. Dyadic interaction processing in the posterior temporal cortex. NeuroImage 198, 296-302.

Walbrin, J., Downing, P., Koldewyn, K., 2018. Neural responses to visually observed social interactions. Neuropsychologia 112, 31-39. 